An Exponential Family of Lorenz Curves

Author(s): José-María Sarabia, Enrique Castillo and Daniel J. Slottje

Source: Southern Economic Journal, Vol. 67, No. 3 (Jan., 2001), pp. 748-756

Published by: Southern Economic Association

Stable URL: http://www.jstor.org/stable/1061463

Accessed: 21/01/2014 12:24

Your use of the JSTOR archive indicates your acceptance of the Terms \& Conditions of Use, available at http://www.jstor.org/page/info/about/policies/terms.jsp

JSTOR is a not-for-profit service that helps scholars, researchers, and students discover, use, and build upon a wide range of content in a trusted digital archive. We use information technology and tools to increase productivity and facilitate new forms of scholarship. For more information about JSTOR, please contact support@ jstor.org. 


\title{
An Exponential Family of Lorenz Curves
}

\author{
José-María Sarabia,* Enrique Castillo, $†$ and Daniel J. Slottjeł
}

\begin{abstract}
A new method for building parametric-functional families of Lorenz curves, generated from an initial Lorenz curve (which satisfies some regularity conditions), is presented. The method is applied to the exponential family since they use the exponential Lorenz curves as their generating curves. Several properties of these families are analyzed, including the population function, inequality measures, and Lorenz orderings. Finally, an application is presented for data from various countries. The family is shown to perform well in fitting the data across countries. The results are very robust across data sources.
\end{abstract}

\section{Introduction}

The purpose of this paper is to introduce a parametric family of Lorenz curves that are obtained by a general method. In a recent paper, Sarabia, Castillo, and Slottje (1999) (SCS) introduced a method that allowed for the building of hierarchies of Lorenz curves when some regularity conditions are satisfied. They introduced the Pareto family, which was found to be a flexible form and which fits actual income distribution data well. This paper introduces another family, the exponential family, which also has interesting characteristics. The exponential family involves more complex estimation with a form that is somewhat less flexible but in return gives a robust performance in fitting actual data across countries, as we will show here. The researcher or policy maker is provided another effective tool in the ongoing effort to quantify, analyze, and understand economic inequality.

The strategy used here is to apply a Lorenz curve hierarchy that contains (as special cases) Lorenz curves derived from this general method. In section 2 we introduce the notation and some necessary background information. The general method is presented in section 3, which starts from an initial Lorenz curve $L_{0}(p)$ (which is called the generating curve) and builds a family with an increasing number of parameters. These in turn can be interpreted in terms of elasticities of $L_{0}(p)$. Also in section 3 we introduce the exponential family of Lorenz curves and discuss some of its properties as population functions and inequality measures and for undertaking Lorenz orderings. In section 4 we present a method for estimating Lorenz curves and apply it to the two families specified previously. Since the goodness of fit is one important criterion in the evaluation of these (and any) models, we use a method due to Gastwirth (1972) and actually incorporate his procedure into the estimation process, as will be clear in section 4 .

\footnotetext{
* Department of Economics, University of Cantabria, Avda. de los Castros s/n, 39005-Santander, Spain

$\dagger$ Department of Applied Mathematics and Computational Sciences, University of Cantabria, Avda. de los Castros $\mathrm{s} / \mathrm{n}, 39005-$ Santander, Spain

‡ Department of Economics, Southern Methodist University, Dallas, TX 75275, USA; E-mail dslottje@mail.smu. edu; corresponding author.

Paper presented at the American Statistical Association meetings, August 9 to 13, 1998, Dallas, Texas. The authors are grateful to the Dirección General de Investigación Científica y Técnica (DGICYT) (project PB96-1261) for partial support of this work. Comments by two anonymous referees have greatly improved the paper. The usual caveat holds.

Received June 1998; accepted March 2000.
} 
An example of an application of our new methodology is presented in section 5. Finally, in section 6 we conclude the paper.

\section{Notation and Previous Results}

In this section we use the Lorenz curve as defined by Gastwirth (1971). That is,

Definition 1. Given a distribution function $F(x)$ with support in the subset of the positive real numbers and with finite expectation $\mu$, we define a Lorenz curve as

$$
L_{F}(p)=\mu^{-1} \int_{0}^{p} F^{-1}(x) d x, \quad 0 \leq p \leq 1,
$$

where

$$
F^{-1}(x)=\sup \{y: F(y) \leq x\} .
$$

A characterization of the Lorenz curve that is attributed to Gaffney and Anstis by Pakes (1981) is given by the following theorem:

THEOREM 1. Assume that $L(p)$ is defined and continuous in the interval $[0,1]$ with second derivative $L^{\prime \prime}(p)$. The function $L(p)$ is a Lorenz curve i.f.f.

$$
L(0)=0, \quad L(1)=1, \quad L^{\prime}\left(0^{+}\right) \geq 0 \quad \text { for } p \in(0,1) \quad L^{\prime \prime}(p) \geq 0 .
$$

Lorenz curves allow establishing a ranking in a set of distributions functions. If two distribution functions have associated Lorenz curves that do not intersect, then they can be ordered without ambiguity in terms of welfare functions that are symmetric, increasing, and quasiconcave (Atkinson 1970; Dasgupta, Sen, and Sarret 1973; Shorrocks 1983). A distribution function $F_{X}(x)$ is said to have less inequality in the Lorenz sense than a distribution function $G_{Y}(y)$ if their Lorenz curves $L_{F}(p)$ and $L_{G}(p)$ satisfy the condition $L_{F}(p) \geq L_{G}(p)$ for all $p$, where the sign $>$ applies for at least one $p \in(0,1)^{\prime}$. In this case we write $X \leq_{L} Y$. From the definition of the Lorenz curve (Eqn. 1), it is evident that the Lorenz partial order is invariant with respect to scale transformations, that is, $X \leq_{L} Y$ i.f.f. $\lambda X \leq_{L} v Y$ for all $\lambda, v>0$.

TheOREM 2. Let $L(p)$ be a Lorenz curve and consider the transformation

$$
L_{\alpha}(p)=p^{\alpha} L(p), \quad \alpha \geq 0 .
$$

Then, if $\alpha \geq 1, L_{\alpha}(p)$ is a Lorenz curve, too. In addition, if $0 \leq \alpha<1$ and $L^{m}(p) \geq 0, L_{\alpha}(p)$ is also a Lorenz curve.

THEOREM 3. If $L(p)$ is a Lorenz curve,

$$
L_{\gamma}(p)=L(p)^{\gamma}, \quad \gamma \geq 1
$$

is a Lorenz curve. Since $L_{\gamma}(p)$ is an increasing convex transform of $L(p)$ and $L_{\gamma}(0)=0$ and $L_{\gamma}(1)=1, L_{\gamma}(p)$ is a Lorenz curve as well. We now present several examples to demonstrate the usefulness of these theorems.

One well-known form of the Lorenz curve is that attributable to Rasche et al. (1980). Other forms are due to Kakwani and Podder (1973) and Kakwani (1980). Rasche et al. (1980) showed 
that Kakwani's Lorenz curve does not satisfy all the requirements for a Lorenz curve. Using our Theorem 1, we find a modified Lorenz curve:

$$
L(p ; a, \beta)=p-a p(1-p)^{\beta}, \quad 0 \leq a \leq 1 ; \quad 0<\beta \leq 1 .
$$

Then, using Theorems 2 and 3, we generate a new family of Lorenz curves:

$$
L_{\mathrm{a}, \alpha, \beta, \gamma}(p)=p^{\alpha+\gamma}\left[1-a(1-p)^{\beta}\right]^{\gamma} ; \quad 0 \leq a \leq 1, \quad \alpha \geq 0, \quad 0<\beta \leq 1, \quad \gamma \geq 1 .
$$

\section{Hierarchical Families of Lorenz Curves}

The previous theorems suggest a method for obtaining hierarchical families of Lorenz curves. Towards this aim, we start with an initial generating Lorenz curve $L_{0}(p)$ and consider the following parametric hierarchy:

$$
\begin{aligned}
L_{1}(p ; \alpha) & =p^{\alpha} L_{0}(p), & & (\alpha \geq 1) \quad \text { or } \quad\left[0 \leq \alpha, 1, L_{0}^{\prime \prime \prime}(p) \geq 0\right] \\
L_{2}(p ; \gamma) & =L_{0}(p)^{\gamma}, & \gamma \geq 1 & \\
L_{3}(p ; \alpha, \gamma) & =p^{\alpha} L_{0}(p)^{\gamma}, & & (\alpha, \gamma \geq 1) \quad \text { or } \quad\left[0 \leq \alpha<1, \gamma \geq 1, L_{0}^{\prime \prime \prime}(p) \geq 0\right] .
\end{aligned}
$$

Families 7 and 8 were obtained using Theorems 2 and 3 and Family 9 arises by combining both results. Note that Families 7 and 8 are ordered with respect to their parameters $\alpha$ and $\gamma$. It is clear that

(a) $L_{1}$ is ordered with respect to $\alpha$ since if $\alpha_{1} \geq \alpha_{2}>0$, then $L_{1}\left(p, \alpha_{1}\right) \leq L_{1}\left(p, \alpha_{2}\right)$.

(b) $L_{2}$ is ordered with respect to $\gamma$ since if $\gamma_{1} \geq \gamma_{2}>0$, then $L_{2}\left(p, \gamma_{1}\right) \leq L_{2}\left(p, \gamma_{2}\right)$.

(c) If $L_{0}(p)=L_{0}(p ; k)$ is ordered with respect to parameter $k$, that is, if $k_{1} \leq k_{2}$, we have

$$
L_{0}\left(p ; k_{1}\right) \leq L_{0}\left(p ; k_{2}\right) \text {. }
$$

Then

(i) If $\alpha_{1} \geq \alpha_{2}$, then

$$
p^{\alpha_{1}} L_{0}\left(p ; k_{1}\right) \leq p^{a_{1}} L_{0}\left(p ; k_{2}\right) \leq p^{a_{2}} L_{0}\left(p ; k_{2}\right) ;
$$

that is, we have new ordering with respect to $\alpha$.

(ii) If $\gamma_{1}>\gamma_{2}$, then

$$
L_{0}^{\gamma_{1}}\left(p ; k_{1}\right) \leq L_{0}^{\gamma_{1}}\left(p ; k_{2}\right) \leq L_{0}^{\gamma_{2}}\left(p ; k_{2}\right)
$$

that is, we have new ordering with respect to $\gamma$.

(d) Combining the previous results, we can also obtain a new ordering for family $L_{3}$. The new parameters that are sequentially incorporated in the hierarchy can be interpreted in terms of the curve elasticities. For example,

$$
\epsilon\left(L_{3} ; p\right)=\alpha+\gamma \epsilon\left(L_{0} ; p\right)
$$

where $\epsilon(L ; p)$ represents the elasticity of $L$.

\section{The Exponential Lorenz Curve Family}

The family we discuss is the exponential Lorenz curve family. This family is generated from the initial Exponential Lorenz curve, 


$$
L_{0}(p ; k)=c_{k}\left(e^{k p}-1\right), \quad 0 \leq p \leq 1,
$$

with $c_{k}^{-1}=e^{k}-1$, which satisfies Theorem 2. This curve is called the exponential Lorenz curve since it is generated from the suitably normalized exponential function $g(p ; k)=\exp (k p)$, $k>0$, and yields the Lorenz curve $L_{0}(p, k)=[g(p ; k)-g(0 ; k)] /[g(1 ; k)-g(0 ; k)]$. This curve has been recently proposed by Chotikapanich (1993) and gives excellent fitting results with grouped data. The model $L_{0}(p, k)$ includes as a particular case the egalitarian model $L(p)$ $=p$. This is a limiting case for $k$ going to zero, that is, $L_{0}(p ; k)=p$. The model $L_{0}(p ; k)$ can also be interpreted as a linear convex combination of an infinite set of potential Lorenz curves, $p^{i}, j=1,2$, with weights decreasing with $i$, that is,

$$
L_{0}(p ; k)=\frac{e^{k p}-1}{e^{k}-1}=\sum_{i=1}^{\infty} w_{i} p^{i}
$$

where

$$
w_{i}=\frac{k^{i}}{i !\left(e^{k}-1\right)}, \quad w_{i} \geq 0, \quad \sum_{i=1}^{\infty} w_{i}=1
$$

In some cases the fit is even better than that associated with some biparametric families. Using previous results again, we can consider the hierarchy of exponential Lorenz curves:

$$
\begin{aligned}
L_{1}(p ; k, \alpha) & =c_{k} p^{\alpha}\left(e^{k p}-1\right) ; & k>0, & \alpha \geq 0 & \\
L_{2}(p ; k, \gamma) & =c_{k, \gamma}\left(e^{k p}-1\right)^{\gamma} ; & k>0, & \gamma \geq 1 & \\
L_{3}(p ; k, \alpha, \gamma) & =c_{k, \gamma} p^{\alpha}\left(e^{k p}-1\right)^{\gamma} ; & k>0, & \alpha \geq 0, & \gamma \geq 1,
\end{aligned}
$$

where $c_{k, \gamma}-\left(e^{k}-1\right)^{-\gamma}$.

\section{Population Functions}

The quantile functions of the exponential hierarchies are given by

$$
\begin{aligned}
X_{0}(p ; k, \mu) & =\mu k c_{k} e^{k p} \\
X_{1}(p ; k, \alpha \mu) & =\mu c_{k}\left[\alpha p^{\alpha-1}\left(e^{k p}-1\right)+k p^{\alpha} e^{k p}\right] \\
X_{2}(p ; k, \gamma \mu) & =\mu \gamma k c_{k, \gamma} e^{k p}\left(e^{k p}-1\right)^{\gamma-1} \\
X_{3}(p ; k, \alpha, \gamma, \mu) & =\mu c_{k, \gamma}\left[\alpha p^{\alpha-1}\left(e^{k p}-1\right)^{\gamma}+k \gamma p^{\alpha} e^{k p}\left(e^{k p}-1\right)^{\gamma-1}\right] .
\end{aligned}
$$

In some particular cases we can obtain closed-form expressions for the distribution functions, as with $L_{0}$. Again we can prove that the distribution function for Equation 20 becomes $F_{0}(x ; k, \mu)=0$ if $x \leq \mu u(k), F_{0}(x ; k, \mu)=1$ if $x \geq \mu \nu(k)$ and

$$
F_{0}(x ; k, \mu)=\frac{1}{k} \log \left[\frac{x}{\mu u(k)}\right] \quad \text { if } \mu u(k) \leq x \leq \mu \nu(k),
$$

where, $u(k)=k /\left(e^{k}-1\right)$ and $v(k)=k e^{k} /\left(e^{k}-1\right)$.

For the remaining families we also can obtain results. For example, for $L_{2}$ with $\gamma=2$, we obtain 


$$
\begin{aligned}
& F_{2}(x ; k, 2, \mu)=\frac{1}{k} \log \left[\frac{1}{2}(1+\sqrt{1+4 x / c})\right] \quad \text { if } 0 \leq x \leq 2 v(k) \mu, \quad k>0, \quad \text { and } \\
& \alpha \geq 0 \quad c=2 k \mu /\left(e^{k}-1\right)^{2} \text { and } \\
& F_{2}(x ; k, 2, \mu)=0 \quad \text { if } x \leq 0 \quad \text { and } \quad F_{2}(x ; k, 2, \mu)=1 \quad \text { if } x \geq 2 v(k) \mu .
\end{aligned}
$$

We present some inequality measures that correspond to these Lorenz curves in the Appendix. We now discuss estimation of these models.

\section{Estimation}

In inequality studies, several types of data are normally utilized: grouped data and micro data. Micro data can consist of a set of individual observations or a set of points on the empirical Lorenz curve, for example, income deciles. The estimation method that is presented here can be used for any of the three types of data. For estimating the parameters of Families 17 to 19 , least squares is the most direct method to be applied. In all cases, we need to minimize a nonlinear function of the parameters. This method presents some well-known problems, such as the need for proving the existence of an absolute minimum and the need from initial values of the estimates for the iterative process to converge. We discuss these problems and propose solutions now.

\section{The Proposed Method}

The merits of parametric methods, as opposed to nonparametric methods, for the construction of indices and inequality measures for income probability distributions with grouped data have recently been discussed by Slottje (1990). Slottje concludes that the indices should be constructed using the parametric method and then the results checked using a nonparametric method. In this sense, Gastwirth's (1972) Gini bounds are nonparametric constraints that should be satisfied by the Gini index of any parametric family of Lorenz curves.

Consequently, any estimation method for the exponential family should lead to parameter values whose Gini indices satisfy Gastwirth's bounds. The usual estimation method consists of minimizing with respect to $\theta$ the sum of squares:

$$
\sum_{i=1}^{n}\left[q_{i}-L\left(p_{i} ; \theta\right)\right]^{2} ; \quad \theta \in \Theta
$$

where $\Theta$ is the set of feasible parameters. Unfortunately, an estimation method based on Equation 26 does not guarantee a Gini satisfying the Gastwirth bounds. An empirical study on this problem has been done by Schader and Schmid (1994), who arrived at conclusions similar to those in Slottje (1990).

One possible solution to this problem consists of incorporating the Gastwirth bounds directly into the programming problem as one more constraint. Thus, we propose to minimize the function Equation 26 subject to

$$
G L \leq 2 \int_{0}^{1}[p-L(p ; \theta)] d p \leq G U
$$


Table 1. Gastwirth Lower Bounds for Different Countries

\begin{tabular}{lc}
\hline Country & Lower Bound to G \\
\hline Brazil & 0.62105 \\
Columbia & 0.54710 \\
Denmark & 0.36215 \\
Finland & 0.46585 \\
India & 0.44925 \\
Indonesia & 0.43575 \\
Japan & 0.30660 \\
Kenya & 0.60635 \\
Malaysia & 0.50345 \\
Netherlands & 0.44210 \\
New Zealand & 0.36580 \\
Norway & 0.35740 \\
Panama & 0.44085 \\
Sri Lanka & 0.40395 \\
Sweden & 0.38205 \\
Tanzania & 0.52615 \\
Tunisia & 0.49645 \\
United Kingdom & 0.35790 \\
Uruguay & 0.49135 \\
\hline
\end{tabular}

where $G L$ and $G U$ are the Gastwirth bounds associated with the set of data $\left(p_{i}, q_{i}\right), i-1, \ldots$, $n$, that is

$$
\begin{aligned}
& G L=1-\sum_{j=1}^{k+1}\left(p_{j}-p_{j-1}\right)\left(q_{j}+q_{j-1}\right) \\
& G U=G L+m^{-1} \sum_{j=1}^{k+1}\left(p_{j}-p_{j-1}\right)^{2}\left(a_{j}-m_{j}\right)\left(m_{j}-a_{j-1}\right)\left(a_{j}-a_{j-1}\right)^{-1}
\end{aligned}
$$

where $p_{0}=q_{0}=0, p_{k+1}=q_{k+1}=1\left[a_{j-1} a_{j}\right]$, are the limits of the income intervals, $m_{j}$ is the mean income of the interval, and $m$ is the overall mean.

Constraint (Eqn. 27) can be incorporated with other alternative estimation methods, as, for example, that proposed in Castillo, Hadi, and Sarabia $(1995,1998)$.

\section{Some Examples}

To illustrate the method proposed here, we apply it to income distribution data on national samples of income recipients across countries. The data are from Shorrocks (1983). The data correspond to figures for cumulated income shares for 19 countries derived from Jain (1975). The 19 countries selected for analysis were chosen because they cover samples with relatively high, middle, and low income groups with varying degrees of inequality.

Using our approach, the Gastwirth lower bounds associated with the different countries are shown in Table 1. As can be seen, the lower bound varies significantly across the countries scrutinized in our study. These should be viewed in light of the overall estimates.

In Tables 2 to 3, we give the parameter estimates and the mean square error (MSE), the 
Table 2. Goodness-of-Fit Measures and Gini Indices Corresponding to Model $L_{1}$

\begin{tabular}{llllll}
\hline Country & \multicolumn{1}{c}{$\kappa$} & \multicolumn{1}{c}{ MAE } & \multicolumn{1}{c}{ MSE } & \multicolumn{1}{c}{ MAXABS } & \multicolumn{1}{c}{ Gini Index } \\
\hline Brazil & 6.11303 & 0.0672026 & 0.00670074 & 0.184059 & 0.677267 \\
Columbia & 4.40909 & 0.0446958 & 0.00353165 & 0.136717 & 0.571024 \\
Denmark & 2.36837 & 0.0103202 & 0.00014356 & 0.0223345 & 0.36215 \\
Finland & 3.27372 & 0.0184473 & 0.000568854 & 0.0520662 & 0.467785 \\
India & 3.23994 & 0.0499807 & 0.00423375 & 0.149348 & 0.46423 \\
Indonesia & 3.15391 & 0.0633097 & 0.00652695 & 0.184605 & 0.455043 \\
Japan & 1.96496 & 0.0146695 & 0.000358936 & 0.0371705 & 0.308185 \\
Kenya & 6.00083 & 0.0766118 & 0.00835894 & 0.202144 & 0.671678 \\
Malaysia & 3.7724 & 0.0343096 & 0.00203517 & 0.102055 & 0.51691 \\
Netherlands & 3.0776 & 0.023943 & 0.000997398 & 0.0704869 & 0.446732 \\
New Zealand & 0.39684 & 0.0132102 & 0.000240519 & 0.0297536 & 0.3658 \\
Norway & 0.33157 & 0.0150283 & 0.000283704 & 0.042860 & 0.3574 \\
Panama & 3.06741 & 0.0247178 & 0.00106703 & 0.0728711 & 0.445611 \\
Sri Lanka & 2.73551 & 0.0172091 & 0.000491571 & 0.0462995 & 0.407594 \\
Sweden & 2.5308 & 0.0146085 & 0.000328096 & 0.03686 & 0.382693 \\
Tanzania & 4.32115 & 0.0635603 & 0.00610748 & 0.173073 & 0.564087 \\
Tunisia & 3.72563 & 0.0276664 & 0.00104612 & 0.0608432 & 0.512564 \\
United Kingdom & 2.34177 & 0.0215614 & 0.000809624 & 0.0627512 & 0.35872 \\
Uruguay & 3.57844 & 0.0159616 & 0.000408415 & 0.0394658 & 0.498539 \\
\hline
\end{tabular}

mean absolute error (MAE), the maximum absolute error (MAXABS) and the Gini index for each country, where

$$
\operatorname{MSE}=\sum_{i=1}^{n}\left[q_{i}-L\left(p_{i} ; \hat{k}, \hat{\alpha}, \hat{\gamma}\right)\right]^{2} / n
$$

is the mean squared error and

Table 3. Goodness-of-Fit Measures and Gini Indices Corresponding to Model $L_{1}$

\begin{tabular}{lcccccc}
\hline Country & $\kappa$ & $\gamma$ & MAE & MSE & MAXABS & Gini Index \\
\hline Brazil & 6.11300 & 1.00019 & 0.0672139 & 0.00670082 & 0.184018 & 0.677324 \\
Columbia & 4.41021 & 1.00000 & 0.0447035 & 0.00353165 & 0.136675 & 0.571111 \\
Denmark & 1.96676 & 1.12001 & 0.0107581 & 0.00016163 & 0.025593 & 0.363753 \\
Finland & 3.26489 & 1.00201 & 0.0184466 & 0.00056943 & 0.052112 & 0.467757 \\
India & 3.24050 & 1.00000 & 0.0499908 & 0.00423375 & 0.149326 & 0.464290 \\
Indonesia & 3.15433 & 1.00000 & 0.0633176 & 0.00652695 & 0.184589 & 0.455088 \\
Japan & 0.08593 & 1.89580 & 0.0342035 & 0.00136918 & 0.054208 & 0.323708 \\
Kenya & 6.00158 & 1.00004 & 0.0766221 & 0.00835896 & 0.202107 & 0.671729 \\
Malaysia & 3.77466 & 1.00004 & 0.0343362 & 0.00203522 & 0.101962 & 0.517135 \\
Netherlands & 3.07786 & 1.00003 & 0.0239475 & 0.00099742 & 0.070474 & 0.446772 \\
New Zealand & 1.47461 & 1.30012 & 0.0137192 & 0.00030297 & 0.035858 & 0.365800 \\
Norway & 1.44674 & 1.28799 & 0.0141477 & 0.00029580 & 0.035039 & 0.357400 \\
Panama & 3.06767 & 1.00001 & 0.0247216 & 0.00106704 & 0.072860 & 0.445644 \\
Sri Lanka & 2.73138 & 1.00148 & 0.0172602 & 0.0049237 & 0.046265 & 0.407800 \\
Sweden & 2.52290 & 1.00157 & 0.0145834 & 0.00032861 & 0.036961 & 0.382464 \\
Tanzania & 4.32084 & 1.00000 & 0.0635580 & 0.00610748 & 0.173085 & 0.564062 \\
Tunisia & 3.72630 & 1.00016 & 0.0276857 & 0.00104624 & 0.060793 & 0.512692 \\
United Kingdom & 2.34042 & 1.00086 & 0.0215970 & 0.00081023 & 0.062703 & 0.358960 \\
Uruguay & 3.57863 & 1.00009 & 0.0159672 & 0.00040845 & 0.039445 & 0.498597 \\
\hline
\end{tabular}




$$
\mathrm{MAE}=\sum_{i=1}^{n}\left|q_{i}-L\left(p_{i}, \hat{k}, \hat{\alpha}, \hat{\gamma}\right)\right| / n
$$

is the mean absolute error. The maximum absolute error is

$$
\text { MAXABS }=\max _{i=1, \ldots, n}\left|q_{i}-L\left(p_{i} ; \hat{k}, \hat{\alpha}, \hat{\gamma}\right)\right|
$$

As can be seen in Tables 2 and 3, across all countries, the first model $\left(L_{1}\right)$ gives lower MAE, MSE, and MAXABS. The order of magnitude of the coefficients, however, is virtually the same. The differences in the measures of goodness of fit are not different until the fourth or fifth decimal place. In sum, $L_{1}$ appears to be a slightly better fitting model than $L_{2}$. The Gini coefficients for the $L_{1}$ and $L_{2}$ models are essentially the same in both cases, but the Ginis are slightly higher in $L_{2}$. In fact, it appears that $L_{1}$ is giving better precision of the model's description of inequality, yet $L_{2}$ yields Gini measures that are more sensitive to inequality. Thus, $L_{2}$ and $L_{1}$ appear to flip-flop across countries with respect to their relative Ginis vis-à-vis their goodness-of-fit measures.

\section{Conclusions and Recommendations}

In this paper we have introduced a new family of Lorenz curves that are generated from the exponential family. Several parameters are incorporated sequentially, keeping the Lorenz character of the resulting families of curves. Several properties of this family are analyzed, and a general estimation method has been proposed that guarantees the existence of unique estimates. The exponential models appear to be very good approximations to actual income distribution data. The results are robust to different data sets for different countries from various parts of the world. Perhaps the most attractive feature of the proffered estimation method is that it is robust. The only cost of this method is some loss of flexibility.

\section{Appendix}

The Gini index of the exponential hierarchy can be expressed in terms of the confluent hypergeometric function, whose integral representation is given by $(b>a)$ :

$$
\begin{gathered}
\frac{\Gamma(b-a) \Gamma(a)}{\Gamma(b)} M(a, b, z)=\int_{0}^{1} e^{z t} t^{a-1}(1-t)^{b-a-1} d t . \\
\Gamma(b-a) \Gamma(a) / \Gamma(b) M(a, b, z)=\int_{0}^{1} e^{z} t^{a-1}(1-t)^{b-a-1} d t .
\end{gathered}
$$

The most important properties of the confluent hypergeometric can be found in Abramowitz and Stegum (1970, p. 503). We have the following theorem.

THEOREM A1. The Gini indices of the exponential hierarchy are given by

$$
\begin{aligned}
G_{0}(k) & =\frac{k\left(e^{k}+1\right)-2\left(e^{k}-1\right)}{k\left(e^{k}-1\right)} \\
G_{1}(k, \alpha) & =1-2 \frac{c^{k}}{\alpha+1}[M(\alpha+1, \alpha+2, k)-1] \\
G_{2}(k, \gamma) & =1-2 c_{k, \gamma} \sum_{i=0}^{\infty} \frac{\Gamma(i-\gamma)\left[e^{k(\gamma-i)}-1\right]}{\Gamma(i+1) \Gamma(-\gamma) k(\gamma-i)} \\
G_{3}(k, \alpha, \gamma) & =1-2 c_{k, \gamma} \sum_{i=0}^{\infty} \frac{\Gamma(i-\gamma)}{\Gamma(i+1) \Gamma(-\gamma)} M[\alpha+1, \alpha+2, k(\gamma-i)],
\end{aligned}
$$

where $\mathrm{B}(\mathrm{)})$ and $\Gamma($ ) are the well-known beta and gamma functions. 
Proof. The index $G_{0}(k)$ is given by Chotikapanich (1993). For $G_{1}(k, \alpha)$, we have

$$
\begin{aligned}
G_{1}(k, \alpha) & =1-2 c_{k} \int_{0}^{1}\left(p^{a} e^{k p}-p^{a}\right) d p=1-2 c_{k}\left[\frac{\Gamma(1) \Gamma(\alpha+1)}{\Gamma(\alpha+2)} M(\alpha+1, \alpha+2, k)-\frac{1}{\alpha+1}\right] \\
& =1-2 \frac{{ }^{c} k}{\alpha+1}[M(\alpha+1, \alpha+2, k)-1],
\end{aligned}
$$

and for the index $G_{2}$, we can write

$$
L_{2}(p ; k, \gamma)=\left(c_{k, \gamma} e^{k p \gamma}\right)^{\gamma}=c_{k, \gamma} e^{k p \gamma}\left(1-e^{-k p}\right)^{\gamma} \sum_{i=0}^{x} \frac{\Gamma(i-\gamma)}{\Gamma(i+1) \Gamma(-\gamma)} e^{-k p i},
$$

and integrating, term by term, we obtain the index $G_{2}$. Finally, the index $G_{3}$ can be obtained in a similar form. $Q E D$.

\section{References}

Abramowitz, Moses, and I. A. Stegum. 1970. Handbook of mathematical functions. New York: Dover Publications.

Atkinson, Anthony B. 1970. On the measurement of inequality. Journal of Economic Theory 2:244-63.

Castillo, Enrique, Ali S. Hadi, and José-María Sarabia. 1995. A method for estimating Lorenz curves. Actas del XXII Congreso Nacional de Estadística e Investigación Operativa: 131-2.

Castillo, Enrique, Ali S. Hadi, and José-María Sarabia. 1998. A method for estimating Lorenz curves. Communications in Statistics, Theory and Methods 27:2037-63.

Chotikapanich, Duankamong. 1993. A comparison of alternative functional forms for the Lorenz curve. Economics Letters 41:129-38.

Dasgupta, P., Amartya K. Sen, and David Starret. 1973. Notes on the measurement of inequality. Journal of Economic Theory 6:180-7.

Gastwirth, Joseph L. 1971. A general definition of the Lorenz curve. Econometrica 39:1037-9.

$\rightarrow$ Gastwirth, Joseph L. 1972. The estimation of the Lorenz curve and Gini index. Review of Economics and Statistics 54: 306-16.

Jain, S. 1975. Size distribution of income. Washington, DC: World Bank Publications.

Kakwani, Nanak C. 1980. On a class of poverty measures. Econometrica 48:437-46.

Kakwani, Nanak C., and Nripesh Podder. 1973. On estimation of Lorenz curves from grouped observations. International Economic Review 14:278-92.

Pakes, Ariel G. 1981. On income distributions and their Lorenz curves. Technical Report, Department of Mathematics, University of Western Australia, Nedlands, WA.

Rasche, R. H., J. Gaffney, A. Koo, and N. Obst. 1980. Functional forms for estimating the Lorenz curve. Econometrica 48:1061-2.

Sarabia, José-María, Enrique Castillo, and Dan J. Slottje. 1999. An ordered family of Lorenz curves. Journal of Econometrics 91:43-60.

Schader, M., and F. Schmid. 1994. Fitting parametric Lorenz curves to grouped income distributions: A critical note. Empirical Economics 19:361-70.

Shorrocks, Anthony F. 1983. Ranking income distributions. Economica 50:3-17.

Slottje, Dan J. 1990. Using grouped data for constructing inequality indices: Parametric vs. non-parametric methods. Economics Letters 32:193-7. 